\title{
Constraining the Polarized Gluon Distribution Function of the Proton with Recent STAR Measurements
}

\author{
Nicholas Lukow*t \\ Temple University, Philadelphia, PA USA \\ E-mail: nicholas.lukowatemple.edu
}

\begin{abstract}
The contribution of the gluon helicity to the spin of the proton is being studied through the use of the unique capability of the Relativistic Heavy Ion Collider (RHIC) to collide polarized protons at $\sqrt{s}=200 \mathrm{GeV}$ and $\sqrt{s}=510 \mathrm{GeV}$. The kinematic coverage of the Solenoidal Tracker At RHIC (STAR) allows access to gluons through quark-gluon and gluon-gluon scattering processes which dominate jet production at low and medium transverse momentum. The polarized gluon distribution function, $\Delta g(x)$, can be constrained through a global analysis by measuring the longitudinal double-spin asymmetry $\left(A_{L L}\right)$ of inclusive jet and di-jet production.

Published inclusive jet results from 2009 at mid-rapidity $(|\eta|<1)$ at $\sqrt{s}=200 \mathrm{GeV}$ have been used in global analyses and suggest a statistically significant non-zero truncated first moment of $\Delta g(x)$ for $x>0.05$. An additional data sample of $43 \mathrm{pb}^{-1}$ has been collected in 2015 . This new data sample is $115 \%$ larger than the previous sample and will improve the precision of $\Delta g(x)$ for $x>0.05$. The status of this new inclusive jet analysis will be presented along with the status of di-jet measurements and previous measurements at $\sqrt{s}=510 \mathrm{GeV}$ which will further constrain $\Delta g(x)$ at lower $x$.
\end{abstract}

XXVII International Workshop on Deep-Inelastic Scattering and Related Subjects - DIS2019 8-12 April, 2019

Torino, Italy

\footnotetext{
${ }^{*}$ Speaker.

${ }^{\dagger}$ For the STAR collaboration
} 


\section{Introduction}

In 1988 results from the European Muon Collaboration showed that the intrinsic spins of the proton constituent quarks contribute little to the proton spin [1]. Recent measurements have shown that the first moment of the polarized quark distribution, $\Delta \Sigma$, is approximately 0.25 [2]. The remaining contribution must then come from the combination of the intrinsic spins of the gluons and the orbital angular momenta of the quarks and gluons. One of the primary goals of the RHIC spin program is to perform high precision measurements to constrain the polarized gluon distribution function, $\Delta g(x)$, of the proton.

The Relativistic Heavy Ion Collider (RHIC), is the only collider capable of colliding polarized proton beams, making it a unique tool for probing the spin structure of the proton. RHIC is capable of colliding polarized proton beams at center-of-mass energies up to $510 \mathrm{GeV}$. The measurements described here were taken with the Solenoidal Tracker At RHIC (STAR). STAR is comprised of many detector subsystems including a Time Projection Chamber (TPC), Barrel and Endcap Electromagnetic Calorimeters (BEMC/EEMC), Vertex Position Detector (VPD), and Zero Degree Calorimeter (ZDC). The TPC provides tracking and covers $|\eta|<1.3$ with full azimuthal coverage. The electromagnetic calorimeters, which cover $-1<\eta<2$ and $2 \pi$ in azimuth, are used to trigger on high momentum particles and to measure the neutral component of jets. The VPD and ZDC are used to measure the relative luminosity [3].

\section{Gluon Polarization Measurements}

At RHIC, $g g$ and $q g$ scatterings dominate jet production at low and medium transverse momentum, making jet production sensitive to the gluon distribution. The polarized gluon distribution can be probed by measuring the longitudinal double-spin asymmetry $\left(A_{L L}\right)$ of jets. Jets are reconstructed using the anti- $k_{t}$ algorithm [4]. Many STAR results have been published, indicating a non-zero gluon polarization [5] [6] [7] [8]. There is still a need to increase the precision of these measurements, and to extend the sampled partonic momentum fraction, $x$, region to lower values.

\subsection{Inclusive Jets}

The first STAR results to show strong evidence of a non-zero gluon polarization were the 2009 inclusive jet $A_{L L}$ results from polarized proton-proton collisions at $\sqrt{s}=200 \mathrm{GeV}$ [5]. The measured $A_{L L}$ was consistently larger than the global fit values predicted at the time, though within the prior uncertainty bands. In 2014, the 2009 inclusive jet results were included in global analyses, and the truncated first moment of $\Delta g(x)$ reported by the NNPDF and DSSV collaborations were $\Delta G=\int_{0.05}^{0.2} \Delta g(x) d x=0.17 \pm 0.06$ [9] and $\Delta G=\int_{0.05}^{1} \Delta g(x) d x=0.19 \pm 0.06$ [10], respectively. 


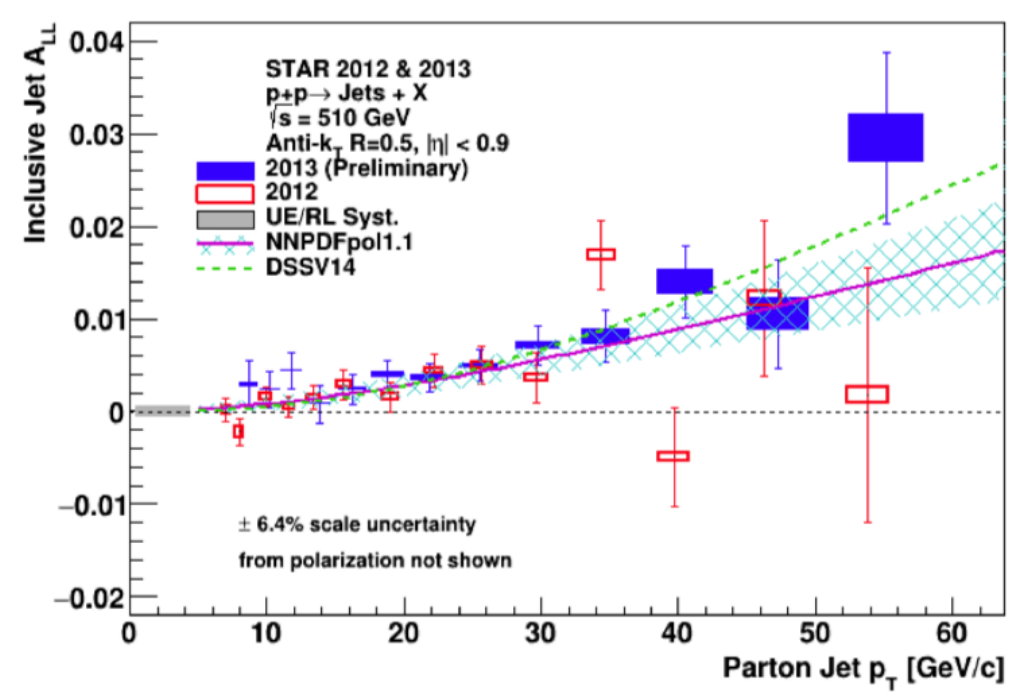

Figure 1: 2012 inclusive jet results [6] compared to preliminary 2013 inclusive jet results. Inclusive jet $A_{L L}$ is plotted vs parton jet $p_{T}$.

The uncertainties at low momentum fraction, $x$, are still large, and more data are needed to constrain $\Delta g(x)$ in that region. In 2012 and 2013 RHIC ran at $\sqrt{s}=510 \mathrm{GeV}$. The higher centerof-mass energy provides sensitivity to lower momentum fractions $(x>0.015)$. The 2012 inclusive jet results were recently submitted for publication [6]. The preliminary 2013 inclusive jet results can be seen in Figure 1, alongside the 2012 inclusive jet results. A Monte Carlo sample embedded in zero bias data is used to quantify systematic uncertainties. With the inclusion of the 2009 results in the newest global fits there is better control over systematic effects such as trigger and reconstruction bias, leading to improved systematic uncertainties.

In 2015 STAR collected more data at $\sqrt{s}=200 \mathrm{GeV}$ to corroborate the results of the 2009 analysis with increased precision. With the increase in sample size, the statistical uncertainties will be further reduced as shown in Figure 2.

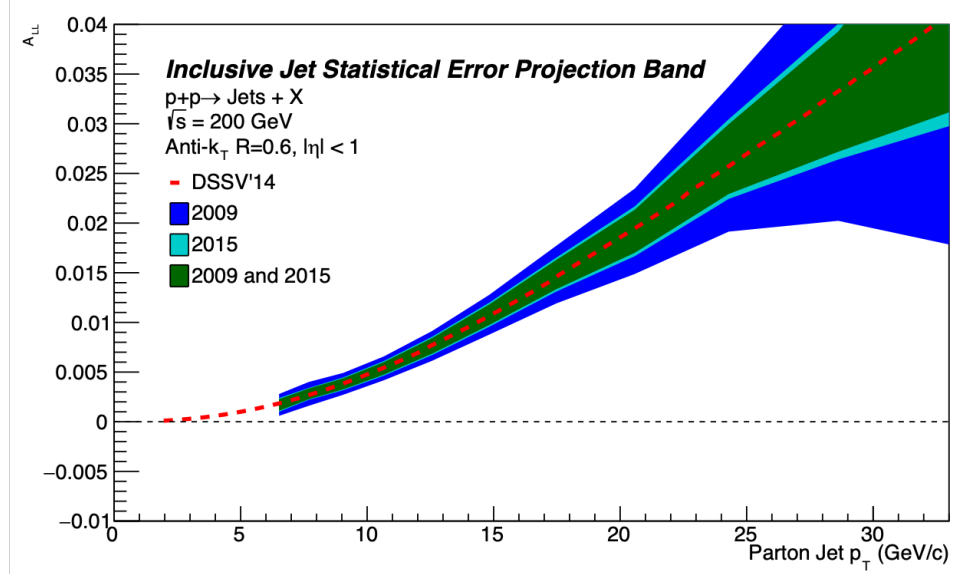

Figure 2: The statistical error band for inclusive jet $A_{L L}$. 


\subsection{Di-Jets}

Di-jets can probe a narrower region in sampled momentum fraction than inclusive jets (Figure 3). The first di-jet results using the 2009 data investigated midrapidity di-jets $(-0.8<\eta<0.8)$ and the results agree well with the 2014 global fits (Figure 4) [7]. Another study using the 2009 data utilized the endcap region of the STAR detector to analyze more forward di-jets. In the forward di-jet analysis at least one jet of the di-jet pair was reconstructed in the endcap $(0.8<\eta<1.8)$ [8]. The results of the forward di-jet analysis agree well with the 2014 global fits except in the most forward topology bin (both jets in the endcap) where the results lie above the the fits but with large uncertainties (Figure 5).

Mid-rapidity di-jet analyses were also performed using the 2012 and $2013 \sqrt{s}=510 \mathrm{GeV}$ data. The preliminary 2013 results can be seen alongside the published 2009 results in Figure 4. The results of the 2012 mid-rapidity di-jet analysis have been submitted for publication and can be seen in [6].

Additional data at $\sqrt{s}=200 \mathrm{GeV}$ were taken in 2015, and another mid-rapidity di-jet analysis is underway using that data set.

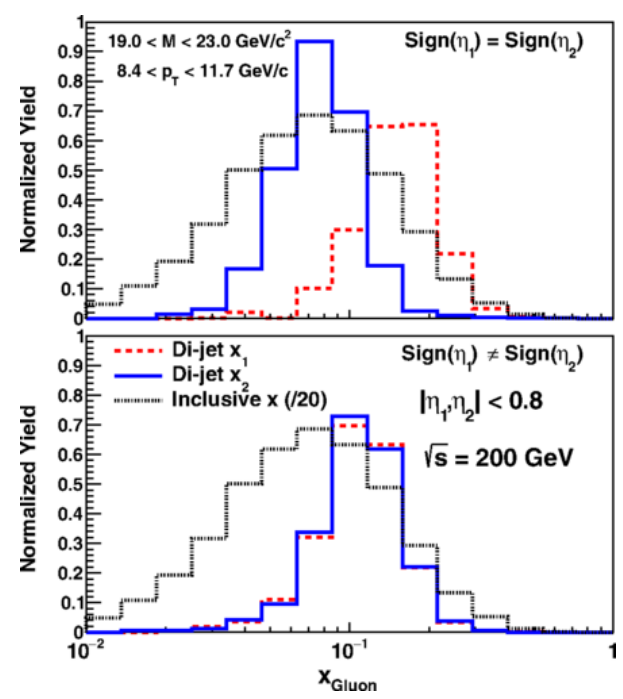

Figure 3: Distributions of the momentum fraction, $x$, of the gluon for two mid-rapidity di-jet configurations using 2009 data. Shown in black is the corresponding distribution for inclusive jets which is considerably wider [7].

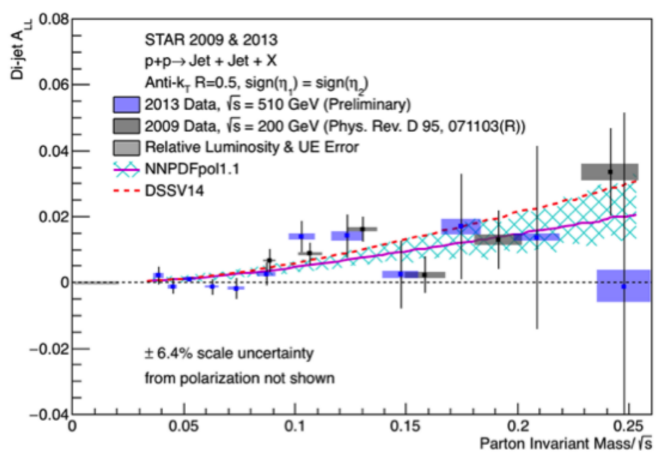

(a) $\operatorname{sign}\left(\eta_{1}\right)=\operatorname{sign}\left(\eta_{2}\right)$

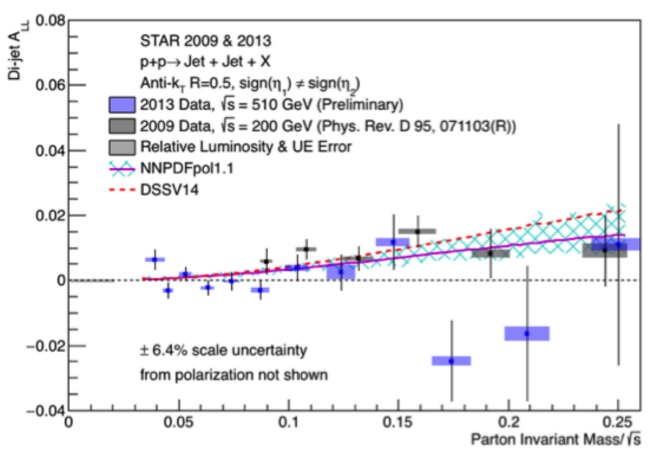

(b) $\operatorname{sign}\left(\eta_{1}\right) \neq \operatorname{sign}\left(\eta_{2}\right)$

Figure 4: 2013 di-jet preliminary results compared ot 2009 di-jet results. Di-jet $A_{L L}$ is plotted vs $M_{\text {invariant }} / \sqrt{s}$. 


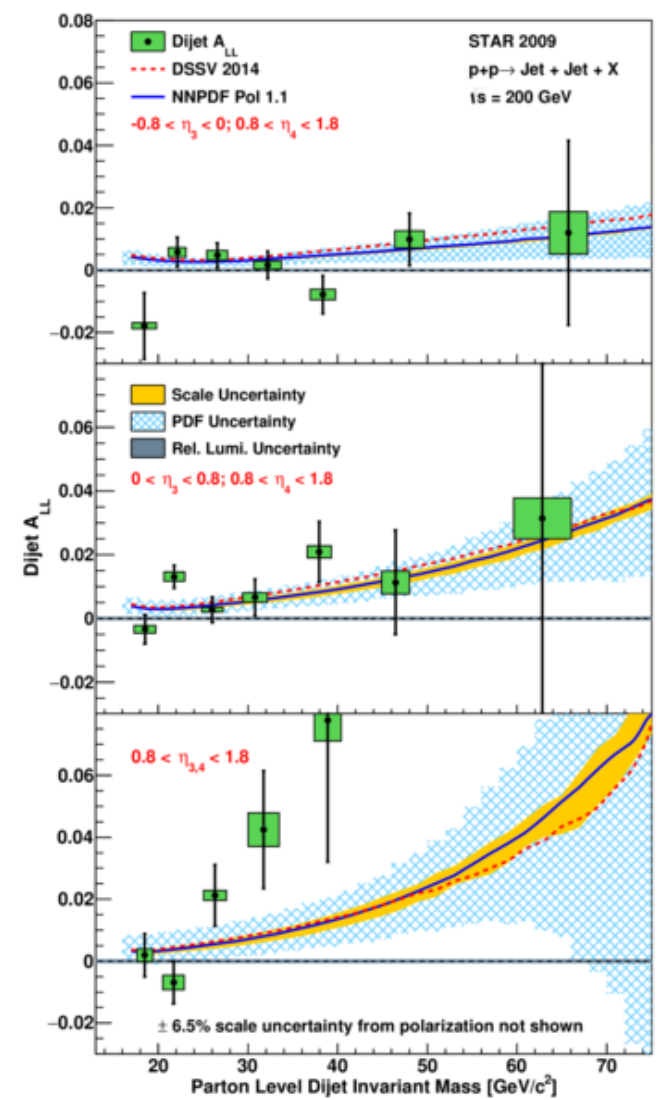

(a) $A_{L L}$ vs di-jet invariant mass for three di-jet (b) configurations.

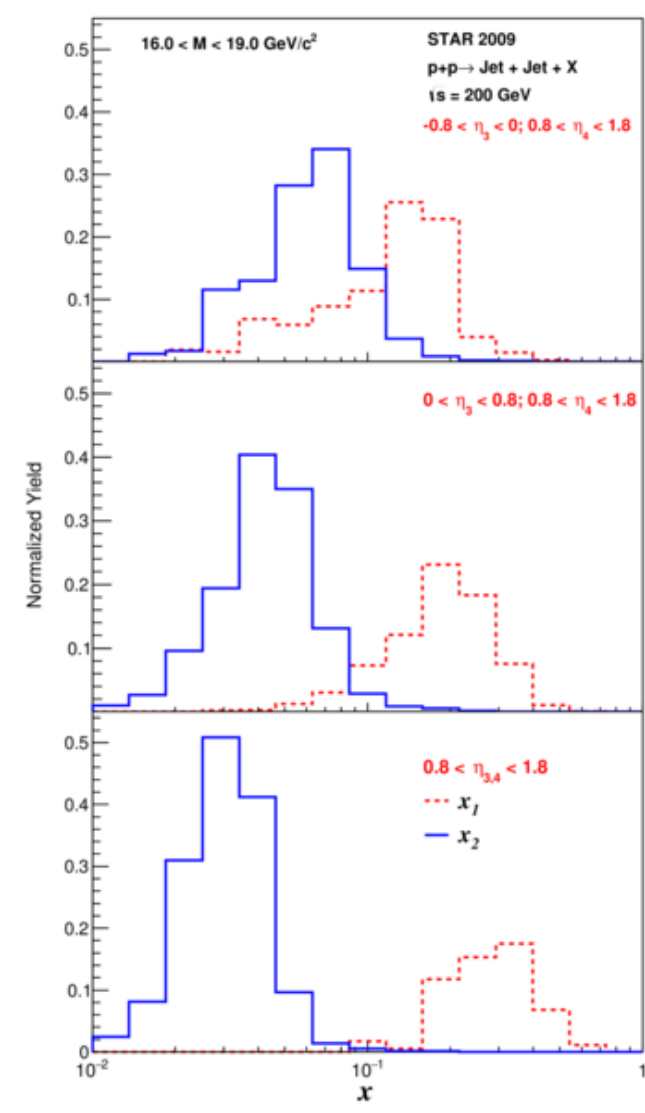

(b) Momentum fraction of the two partons for three di-jet configurations.

Figure 5: 2009 forward di-jet results [8].

\section{Summary}

The STAR collaboration has performed measurements of inclusive jet and di-jet $A_{L L}$ at $\sqrt{s}=$ 200 and $510 \mathrm{GeV}$. The STAR 2009 inclusive jet results were the first to show evidence of a nonzero gluon polarization. All the results are consistent with each other and with the most recent global analyses, NNPDFpol1.1 [9] and DSSV'14 [10]. The DSSV collaboration recently included all the results of analyses using the 2009 data set in a Monte Carlo reweighting analysis, improving the uncertainty in the high- $x$ region [11]. The need for more data at lower values of $x$ provide motivation for the STAR Forward Upgrade and a future EIC facility [12].

\section{References}

[1] J. Ashman et al. [European Muon Collaboration] Phys. Lett. B 206 (2), 364 (1988).

[2] C. A. Aidala, S. D. Bass, D. Hasch and G. K. Mallot (2013) [hep-ph/1209.2803v2].

[3] K. Ackermann et al. [STAR Collaboration], Nucl. Instr. \& Meth. A 499624 (2003).

[4] M. Cacciari, G. P. Salam, and G. Soyez, JHEP 04, 063 (2008). 
[5] L. Adamczyk et al. [STAR Collaboration], Phys. Rev. Lett. 115, 092002 (2015).

[6] J. Adam et al. [STAR Collaboration] (2019) [hep-ex/1906.02740v1].

[7] L. Adamczyk et al. [STAR Collaboration], Phys. Rev. D 95, 071103(R) (2017).

[8] J. Adam et al. [STAR Collaboration], Phys. Rev. D 98, 032011 (2018)

[9] E. R. Nocera et al. [NNPDF Collaboration], Nucl. Phys. B 887, 276 (2014).

[10] D. de Florian, R. Sassot, M. Stratmann and W. Vogelsang, Phys. Rev. Lett. 113, 012001 (2014).

[11] D. de Florian, G. A. Lucero, R. Sassot, M. Stratmann and W. Vogelsang (2019) [hep-ph/1902.10548v1].

[12] A. Accardi et al., BNL-98815-2012-JA; JLAB-PHY-12-1652 (2014) [nucl-ex/1212.1701]. 